\title{
Non-Abelian Chern-Simons Particles and their Quantization*
}

\author{
Dongsu Bak and R. Jackiw \\ Center for Theoretical Physics, Laboratory for Nuclear Science, and Department of Physics \\ Massachusetts Institute of Technology, Cambridge, Massachusetts 02139 U.S.A. \\ So-Young Pi \\ Department of Physics, Boston University, Boston, Massachusetts 02215 U.S.A.
}

\begin{abstract}
A many-body Schrödinger equation for non-Abelian Chern-Simons particles is obtained from both point-particle and field-theoretic pictures. We present a particle Lagrangian and a field theoretic Lagrange density, and discuss their properties. Both are quantized by the symplectic method of Hamiltonian reduction. An $N$-body Schrödinger equation for the particles is obtained from both starting points. It is shown that the resulting interaction between particles can be replaced by nontrivial boundary conditions. Also, the equation is compared with the one given in the literature.
\end{abstract}

Submitted to: Physical Review D

CTP \# 2276

hep-th/9402057

January 1994

\footnotetext{
* This work is supported in part by funds provided by the U. S. Department of Energy (D.O.E.) under contracts \#DE-AC02-76ER03069 and \#DE-AC02-89ER40509, as well as in part by the National Science Foundation under contracts \#INT-910559 and \#INT-910653
} 


\section{Introduction}

Systems of point particles carrying non-Abelian charge in interaction with a non-Abelian gauge field have been under investigation for over two decades. ${ }^{1}$ Recently, such a model in $2+1$ dimensions with Chern-Simons kinetic action has gained attention owing to its peculiar long range interactions. ${ }^{2}$

In general, particles interacting via the Abelian Chern-Simons term acquire fractional statistics, ${ }^{3}$ and are called anyons. ${ }^{4}$ Anyons play a role in the fractional quantum Hall effect ${ }^{5}$ and perhaps also in high temperature superconductivity. ${ }^{6}$

A system of non-relativistic point particles with Abelian Chern-Simons interactions has been previously discussed in [7] and [8]. Non-relativistic quantum dynamics can be equivalently described by a point particle Lagrangian or by a field theory; upon quantization both lead to an $N$-body Schrödinger equation, with the Aharonov-Bohm (Ehrenberg-Siday) interaction. These two approaches are pursued respectively in Refs. [7] and [8]. Moreover when the field theoretical description is analyzed classically, self-dual solitons are found. ${ }^{9}$ (Soliton solutions exist when a quartic self-interaction is included. Quantum mechanically this corresponds to a $\delta$-function hard-core interparticle potential, whose only effect is to regularize the Chern-Simons interaction, protecting its classical conformal invariance ${ }^{10}$ against quantum anomalies. ${ }^{11}$ )

In this paper we extend our previous investigations to the non-Abelian case. Much has already been established on this problem. Non-Abelian self-dual solitons have been found in the corresponding non-relativistic field theory, ${ }^{12}$ which however has never been quantized. The $N$-body Schrödinger equation has been posited on the basis of non-Abelian braid group investigations, ${ }^{13}$ but without a derivation from first principles. A systematic derivation from a particle Lagrangian for the $S U(n)$ group was given subsequently. ${ }^{14}$ In these works use is made of the Knizhnik-Zamolodchikov connection ${ }^{15}$ - a non-Hermitian choice of gauge, which requires a non-trivial compensating measure in the normalization integral.

In Section II, we reconsider the derivation of the Schrödinger equation from a particle Lagrangian. We use the symplectic methods of Hamiltonian reduction ${ }^{16}$ and can accommodate any non-Abelian gauge group, provided it is equipped with an invariant metric. Furthermore, we choose a real gauge and dispense with the complex Knizhnik-Zamolodchikov connection. Both distinguishable and identical particles are considered.

In Section III, we follow the alternative route to the Schrödinger equation, by beginning with a field theoretic Lagrange density. Again using the symplectic method and constructing the $N$-particle state as an eigenstate of the number operator, allows deriving the $N$-body equation, which coincides with the one obtained in Section II for identical particles.

In Section IV, the Schrödinger equation is analyzed. We show that the interaction between the particles may be replaced by a boundary condition and is equivalent to the one given by the Knizhnik-Zamolodchikov connection. 
An Appendix is devoted to a symplectic quantization of an arbitrary Lie algebra, possessing an invariant metric. This is accomplished without explicit passage to Darboux (canonical) variables.

\section{Non-Abelian Chern-Simons particles for an arbitrary group}

The non-Abelian charge carried by a point particle may be viewed as a classical internal degree of freedom that produces a non-Abelian gauge field. Upon quantizing this degree of freedom, the charge operator acquires a spectrum, which leads to a multiplet structure of the particle state.

Let us begin with a classical description on the level of equations of motions. Since the source produces a non-Abelian gauge field, we expect that a field equation relates the field to the source.

$$
\begin{aligned}
& \left(D_{\mu} F^{\mu \nu}\right)^{a}+\frac{\kappa}{2} \epsilon^{\nu \alpha \beta}\left(F_{\alpha \beta}\right)^{a}=j^{\nu} I^{a} \\
& D_{\mu} \equiv \partial_{\mu}+\left[A_{\mu}, \cdots\right]
\end{aligned}
$$

A Chern-Simons term with strength $\kappa$, is included because we are considering a $(2+1)$ dimensional theory. Here $j^{0}(t, \mathbf{r})=\delta(\mathbf{r}-\mathbf{q}(t))$ and $\mathbf{j}(t, \mathbf{r})=\dot{\mathbf{q}}(t) \delta(\mathbf{r}-\mathbf{q}(t))$ comprise the conserved particle current density, $j^{\mu}$, for a particle located in the plane at $\mathbf{q}(t)$ and $I^{a}(t)$ is the non-Abelian charge. [Henceforth, we supress a common time argument in all dynamical quantities.] Since the left side of (1) is covariantly conserved, so must be the right side. This puts an additional condition on $I$ :

$$
\begin{aligned}
D_{\mu}\left(j^{\mu} I\right) & =\left(\partial_{\mu} j^{\mu}\right) I+j^{\mu} D_{\mu} I \\
& =\delta(\mathbf{r}-\mathbf{q}) D_{q} I=0
\end{aligned}
$$

with $D_{q} \equiv \partial_{t}+\left[A_{q}, \cdots\right], A_{q} \equiv A_{0}-\dot{\mathbf{q}} \cdot \mathbf{A}, A_{\mu} \equiv A_{\mu}^{a} T_{a}, I \equiv I^{a} T_{a}$, and the generators $T_{a}$ (in an arbitrary representation) satisfy $\left[T_{a}, T_{b}\right]=f_{a b}{ }^{c} T_{c}$. Our space-time metric tensor is $\operatorname{diag}(1,-1,-1)$. The force law, a non-Abelian generalization of the Lorentz force, is given by

$$
M \ddot{\mathbf{q}}=I_{a}(\mathbf{E}+\dot{\mathbf{q}} \times B)^{a}
$$

where the non-Abelian electric and magnetic fields are, respectively, $E^{i a}=F^{i o a}, \epsilon^{i j} B^{a}=$ $-F^{i j a}$ and $\left(\dot{\mathbf{q}} \times B^{a}\right)^{i} \equiv \epsilon^{i j} \dot{q}^{j} B^{a}$. Eqs. (1), (2) and (3) are the celebrated Wong equations. ${ }^{1}$

Notice that presentation of the Lorentz force equation (3) requires lowering the group index on the non-Abelian charge. For semi-simple groups this is accomplished with the non-singular Cartan-Killing metric $g_{a a^{\prime}}=-\frac{1}{2} f_{a b}{ }^{c} f_{a^{\prime} c}{ }^{b}$. For non-semi-simple groups, the Cartan-Killing metric is singular, so we must assume that there exists another invariant nonsingular form on the Lie algebra which can serve as a metric. This is the only assumption we need to make about the structure of the gauge group. 
Under a local gauge transformation by a group element $U$, the gauge potential $A$ and the particle coordinate $\mathbf{q}$ transform as

$$
A_{\mu} \rightarrow U^{-1} A_{\mu} U+U^{-1} \partial_{\mu} U, \quad \mathbf{q} \rightarrow \mathbf{q}
$$

To preserve gauge covariance of the equations of motion, the charge $I$ must be transform covariantly.

$$
I \rightarrow U^{-1} I U
$$

Since we are interested only in the Chern-Simons kinetic term, we drop the non-Abelian Maxwell (Yang-Mills) term in (1). Then the equations for the $N$-particle system read

$$
\begin{gathered}
\frac{\kappa}{2} \epsilon^{\nu \alpha \beta}\left(F_{\alpha \beta}\right)^{a}=\sum_{k=1}^{N} j_{k}^{\nu} I_{k}^{a} \\
\dot{I}_{k}^{a}+f_{b c}{ }^{a}\left[A_{0}\left(\mathbf{q}_{k}\right)-\dot{\mathbf{q}} \cdot \mathbf{A}\left(\mathbf{q}_{k}\right)\right]^{b} I_{k}^{c}=0 \\
M_{k} \ddot{\mathbf{q}}_{k}=I_{k a}\left[\mathbf{E}\left(\mathbf{q}_{k}\right)+\dot{\mathbf{q}}_{k} \times B\left(\mathbf{q}_{k}\right)\right]^{a}
\end{gathered}
$$

Eqs. (6.a-c) are the Euler-Lagrange equations for the Lagrangian

$$
\begin{aligned}
L & =\frac{1}{2} \sum M_{k} \dot{\mathbf{q}}_{k}^{2}+2 \sum \operatorname{tr} K_{k} g_{k}^{-1} \dot{g}_{k}+2 \int d^{2} r \sum \operatorname{tr} j_{k}^{\nu} I_{k} A_{\nu}\left(\mathbf{q}_{k}\right) \\
& -\kappa \int d^{2} r \epsilon^{\alpha \beta \gamma} \operatorname{tr}\left(A_{\alpha} \partial_{\beta} A_{\gamma}+\frac{2}{3} A_{\alpha} A_{\beta} A_{\gamma}\right)
\end{aligned}
$$

Here "tr" stands for the trace, with $T_{a}$ normalized by $\operatorname{tr} T_{a} T_{b}=-\frac{1}{2} g_{a b}$; when the trace is not available, it is replaced by a non-degenerate bilinear form on the Lie algebra, which also defines the metric. The $K_{k}$ are time-independent elements (in $N$ copies) of the Lie algebra (one for each $k$ ) and $I_{k}$ is defined as

$$
I_{k}=g_{k} K_{k} g_{k}^{-1}
$$

where $g_{k}$ are time-dependent group elements, responding to a gauge transformation by $g_{k} \rightarrow$ $g_{k}^{\prime}=U^{-1} g_{k}$; as a consequence the middle two terms in (7) combine to $2 \sum \operatorname{tr} K_{k} g_{k}^{-1}\left(\partial_{t}+A_{q}\right) g_{k}$. The Euler-Lagrange equations (6.a-c) are derived by varying $A_{\mu}, g_{k}$ and $\mathbf{q}_{k}$.

Upon introducing canonical momenta for the positions $\mathbf{q}_{k}$,

$$
\mathbf{p}_{k} \equiv \frac{\partial L}{\partial \dot{\mathbf{q}}_{k}}=M_{k} \dot{\mathbf{q}}_{k}+I_{k a} \mathbf{A}^{a}\left(\mathbf{q}_{k}\right)
$$


and by performing a Legendre transformation, the Lagrangian is converted into a first-order expression.

$$
\begin{aligned}
L & =\sum\left[\mathbf{p}_{k} \cdot \dot{\mathbf{q}}_{k}+2 \operatorname{tr} K_{k} g_{k}^{-1} \dot{g}_{k}\right]+\kappa \int d^{2} r \dot{A}_{1 a} A_{2}^{a} \\
& -\sum \frac{1}{2 M_{k}}\left(\mathbf{p}_{k}-I_{k a} \mathbf{A}^{a}\left(\mathbf{q}_{k}\right)\right)^{2} \\
& -\int d^{2} r\left\{A_{0}^{a}\left(\kappa B_{a}+\rho_{a}\right)+\frac{\kappa}{2}\left[\partial_{t}\left(A_{1}^{a} A_{2 a}\right)+\partial_{1}\left(A_{2}^{a} A_{0 a}\right)-\partial_{2}\left(A_{1}^{a} A_{0 a}\right)\right]\right\} \\
& \rho^{a} \equiv \sum I_{k}^{a} \delta\left(\mathbf{r}-\mathbf{q}_{\mathbf{k}}\right)
\end{aligned}
$$

Under a gauge transformation the dynamical variable $\mathbf{p}_{k}$ transforms as

$$
\mathbf{p}_{k} \rightarrow \mathbf{p}_{k}+2 \operatorname{tr} I_{k} \nabla U\left(\mathbf{q}_{k}\right) U^{-1}\left(\mathbf{q}_{k}\right)
$$

and the transformed Lagrangian differs by total derivative

$$
L \rightarrow L+\kappa \int d^{2} r \epsilon^{\alpha \beta \gamma}\left[\partial_{\alpha} \operatorname{tr}\left(\partial_{\beta} U U^{-1} A_{\gamma}\right)+\frac{1}{3} \operatorname{tr} U^{-1} \partial_{\alpha} U U^{-1} \partial_{\beta} U U^{-1} \partial_{\gamma} U\right]
$$

The gauge invariance of the quantum theory requires a quantized coupling constant $4 \pi \kappa \in \mathbf{Z}$, when the gauge group is compact and non-Abelian. ${ }^{17}$

To quantize the system, we follow symplectic reduction method. ${ }^{16}$ Note that the Lagrangian in (11) is constrained by Lagrange multipliers $A_{0}^{a}$ and the corresponding constraints are

$$
B^{a}+\frac{1}{\kappa} \rho^{a}=0
$$

We solve the constraints by expressing $A_{2}$ in term of other quantities.

$$
A_{2}^{a}(\mathbf{r})=\int d^{2} r^{\prime} \mathcal{M}_{b}^{a}\left(\mathbf{r}, \mathbf{r}^{\prime}\right)\left[\partial_{2}^{\prime} A_{1}^{b}\left(\mathbf{r}^{\prime}\right)+\frac{1}{\kappa} \rho^{b}\left(\mathbf{r}^{\prime}\right)\right]
$$

where $\mathcal{M}^{a}{ }_{b}\left(\mathbf{r}, \mathbf{r}^{\prime}\right)$ is defined through the following relations,

$$
\begin{aligned}
& \int d^{2} r^{\prime} \mathcal{M}^{-1}{ }_{b}{ }_{b}\left(\mathbf{r}, \mathbf{r}^{\prime}\right) c^{b}\left(\mathbf{r}^{\prime}\right)=\partial_{1} c^{a}(\mathbf{r})+f_{b c}{ }^{a} A_{1}^{b}(\mathbf{r}) c^{c}(\mathbf{r}) \\
& \int d^{2} r^{\prime} \mathcal{M}^{a}{ }_{b}\left(\mathbf{r}, \mathbf{r}^{\prime}\right) \mathcal{M}^{-1}{ }_{c}^{b}\left(\mathbf{r}^{\prime}, \mathbf{r}^{\prime \prime}\right)=\delta\left(\mathbf{r}-\mathbf{r}^{\prime \prime}\right) \delta_{c}^{a}
\end{aligned}
$$

Inserting (15) into (11), we are left with

$$
\begin{aligned}
L & =\sum\left[\mathbf{p}_{k} \cdot \dot{\mathbf{q}}_{k}+2 \operatorname{tr} K_{k} g_{k}^{-1} \dot{g}_{k}\right]+\kappa \int d^{2} r \dot{A}_{1 a}(\mathbf{r}) \int d^{2} r^{\prime} \mathcal{M}_{b}^{a}\left(\mathbf{r}, \mathbf{r}^{\prime}\right)\left[\partial_{2}^{\prime} A_{1}^{b}\left(\mathbf{r}^{\prime}\right)+\frac{1}{\kappa} \rho^{b}\left(\mathbf{r}^{\prime}\right)\right] \\
& -\sum \frac{1}{2 M_{k}}\left[\left(p_{k}^{x}+I_{k a} A_{1}^{a}\left(\mathbf{q}_{k}\right)\right)^{2}+\left(p_{k}^{y}+I_{k a} \int d^{2} r^{\prime} \mathcal{M}^{a}{ }_{b}\left(\mathbf{q}_{k}, \mathbf{r}^{\prime}\right)\left[\partial_{2}^{\prime} A_{1}^{b}\left(\mathbf{r}^{\prime}\right)+\frac{1}{\kappa} \rho^{b}\left(\mathbf{r}^{\prime}\right)\right]\right)^{2}\right]
\end{aligned}
$$


where we ignore irrelevant total derivative terms. While the constraints have been eliminated, the symplectic one-form in the Lagrangian (17) is still not of a desired canonical form. To achieve that, let us make a Darboux transformation. We introduce new (primed) variables with the relations,

$$
\begin{aligned}
g_{k} & =U g_{k}^{\prime} \\
\mathbf{A} & =U \mathbf{A}^{\prime} U^{-1}-U \nabla U^{-1} \\
\mathbf{p}_{k} & =\mathbf{p}_{k}^{\prime}-2 \operatorname{tr} I_{k} \nabla U U^{-1}
\end{aligned}
$$

where $A_{2}$ is given by (15) and $U$ will be specified presently. The Lagrangian (17) becomes

$$
\begin{aligned}
L & =\sum\left[\mathbf{p}_{k}^{\prime} \cdot \dot{\mathbf{q}}_{k}+2 \operatorname{tr} K_{k} g_{k}^{\prime-1} \dot{g}_{k}^{\prime}\right]+\kappa \int d^{2} r \dot{A}_{1 a}^{\prime} A_{2}^{\prime a} \\
& -\sum \frac{1}{2 M_{k}}\left[\mathbf{p}_{k}^{\prime}-I_{k a}^{\prime} \mathbf{A}^{\prime a}\left(\mathbf{q}_{k}\right)\right]^{2}-\frac{\kappa}{3} \int d^{2} r \epsilon^{\alpha \beta \gamma} \operatorname{tr} U^{-1} \partial_{\alpha} U U^{-1} \partial_{\beta} U U^{-1} \partial_{\gamma} U
\end{aligned}
$$

where irrelevant total derivative terms are again ignored.

In the Lagrangian (19), $A_{2}^{\prime}$ may be given in two different ways. First, using (15) and (18), $A_{2}^{\prime}$ is

$$
A_{2}^{\prime}=U^{-1} \int d^{2} r^{\prime} T_{a} \mathcal{M}_{b}^{a}\left(\mathbf{r}, \mathbf{r}^{\prime}\right)\left[\partial_{2} A_{1}^{b}\left(\mathbf{r}^{\prime}\right)+\frac{1}{\kappa} \rho^{b}\left(\mathbf{r}^{\prime}\right)\right] U+U^{-1} \partial_{2} U
$$

On the other hand, one may rewrite (14) in terms of the transformed quantities:

$$
\begin{aligned}
& \partial_{1} A_{2}^{\prime a}-\partial_{2} A_{1}^{\prime a}+f_{b c}{ }^{a} A_{1}^{\prime b} A_{2}^{\prime c}-\frac{1}{\kappa} \sum I_{k}^{\prime a} \delta\left(\mathbf{r}-\mathbf{q}_{\mathbf{k}}\right)=0 \\
& I_{k}^{\prime} \equiv g_{k}^{\prime} K_{k} g_{k}^{\prime-1}
\end{aligned}
$$

Eq. (21) determines an expression for $A_{2}^{\prime}$ that apparently differs from (20); of course the two must agree.

To proceed, take $U$ to satisfy the following relation

$$
A_{1}^{\prime}=U^{-1} A_{1} U+U^{-1} \partial_{1} U=0
$$

From Eq. (21), we have

$$
A_{2}^{\prime}=\frac{1}{\kappa \partial_{1}} \sum I_{k}^{\prime} \delta\left(\mathbf{r}-\mathbf{q}_{\mathbf{k}}\right)=\frac{1}{\kappa} \sum G\left(\mathbf{r}-\mathbf{q}_{\mathbf{k}}\right) I_{k}^{\prime}
$$

where the operator $\frac{1}{\partial_{1}}$ is represented by $\int d^{2} r^{\prime} G\left(\mathbf{r}-\mathbf{r}^{\prime}\right)$. Inserting this into (19), we finally get the Darboux transformed Lagrangian,

$$
\begin{aligned}
L & =\sum_{k}\left[\mathbf{p}_{k}^{\prime} \cdot \dot{\mathbf{q}}_{k}+2 \operatorname{tr} K_{k} g_{k}^{\prime-1} \dot{g}_{k}^{\prime}\right] \\
& -\sum_{k} \frac{1}{2 M_{k}}\left[\left(p_{k}^{\prime x}\right)^{2}+\left(p_{k}^{\prime y}+\frac{1}{\kappa} \sum_{l} G\left(\mathbf{q}_{k}-\mathbf{q}_{l}\right) I_{k}^{\prime a} I_{l a}^{\prime}\right)^{2}\right] \\
& -\frac{\kappa}{3} \int d^{2} r \epsilon^{\alpha \beta \gamma} \operatorname{tr} U^{-1} \partial_{\alpha} U U^{-1} \partial_{\beta} U U^{-1} \partial_{\gamma} U
\end{aligned}
$$


The last term in (24) is a topological surface term, involving variables that decouple from the rest of the Lagrangian, so we drop it, and the Darboux transformed Lagrangian is simply

$$
\begin{aligned}
L & =\sum_{k}\left[\mathbf{p}_{k} \cdot \dot{\mathbf{q}}_{k}+2 \operatorname{tr} K_{k} g_{k}^{-1} \dot{g}_{k}\right] \\
& -\sum_{k} \frac{1}{2 M_{k}}\left[\left(p_{k}^{x}\right)^{2}+\left(p_{k}^{y}+\frac{1}{\kappa} \sum_{l} G\left(\mathbf{q}_{k}-\mathbf{q}_{l}\right) I_{k}^{a} I_{l a}\right)^{2}\right]
\end{aligned}
$$

where primes have been suppressed. In order to quantize the Lagrangian in (25), we must still put $2 \operatorname{tr} \sum K_{k} g_{k}^{-1} \dot{g}_{k}$ into canonical form. However, as we show in the Appendix, one may obtain the required commutation relations [Eq. (28.b) below] without the complicated passage to Darboux variables. So we remain with (25), but a few comments are in order. As mentioned earlier, special care should be taken in choosing $U$ [as a solution to (22)] so that (20) be consistent with (23). As we show in Section IV, the consistency of the Schrödinger equation puts a restriction on $G$. Our consistent choice is

$$
G(\mathbf{r})=\frac{1}{2} \delta(y) \epsilon(x)
$$

The self interaction can be ignored; this is equivalent to setting $G(0) \equiv 0$. Hence, we conclude that an accurate expression of the connection for the $k$-th variable is

$$
\mathcal{G}_{k}=\frac{1}{\kappa} \sum_{l \neq k} \mathbf{G}\left(\mathbf{q}_{k}-\mathbf{q}_{l}\right) I_{k}^{a} I_{l a}
$$

with $G_{x}=0$ and $G_{y}(\mathbf{r})=G(\mathbf{r})$.

Now, it is clear that the fundamental commutation relations are

$$
\begin{gathered}
{\left[q_{k}^{i}, p_{l}^{j}\right]=i \delta_{k l} \delta^{i j}} \\
{\left[I_{k a}, I_{l b}\right]=-i f_{a b}{ }^{c} I_{k c} \delta_{k l}}
\end{gathered}
$$

and the Hamiltonian of the system is

$$
H=\sum_{k} \frac{1}{2 M_{k}}\left[\mathbf{p}_{k}-\frac{1}{\kappa} \sum_{l \neq k} \mathbf{G}\left(\mathbf{q}_{k}-\mathbf{q}_{l}\right) I_{k}^{a} I_{l a}\right]^{2}
$$

[Eqs. (28.b) are derived in the Appendix.] The Hilbert space may be realized in a wavefunction representation, $\phi_{m_{1}, \ldots, m_{N}}\left(\mathbf{q}_{1}, \ldots, \mathbf{q}_{N}\right)$ and each operator is realized by

$$
\mathbf{q}_{k} \phi_{m_{1}, \ldots, m_{k}, \ldots, m_{N}}=\mathbf{q}_{k} \phi_{m_{1}, \ldots, m_{k}, \ldots, m_{N}}
$$




$$
\begin{aligned}
\mathbf{p}_{k} \phi_{m_{1}, \ldots, m_{k}, \ldots, m_{N}} & =\frac{\partial}{i \partial \mathbf{q}_{k}} \phi_{m_{1}, \ldots, m_{k}, \ldots, m_{N}} \\
\left(I_{k}^{a} \phi\right)_{m_{1}, \ldots, m_{k}, \ldots, m_{N}} & =\left(I_{k}^{a}\right)_{m_{k} m_{k}^{\prime}} \phi_{m_{1}, \ldots, m_{k}^{\prime}, \ldots, m_{N}}
\end{aligned}
$$

where $\left(I_{k}^{a}\right)_{m_{k} m_{k}^{\prime}}$ is a corresponding matrix representation of the commutation relations (28.b) (i.e. a Lie group algebra). Hence the Schrödinger equation is simply

$$
\begin{aligned}
& i \partial_{t} \phi\left(\mathbf{q}_{1}, \ldots, \mathbf{q}_{N}\right) \\
& \quad=-\sum_{k} \frac{1}{2 M_{k}}\left(\nabla_{k}-\frac{i}{\kappa} \sum_{l \neq k} \mathbf{G}\left(\mathbf{q}_{k}-\mathbf{q}_{l}\right) I_{k}^{a} I_{l a}\right)^{2} \phi\left(\mathbf{q}_{1}, \ldots, \mathbf{q}_{N}\right)
\end{aligned}
$$

In case the particles are identical, the exchange symmetry should be imposed on the wave-function. Namely, we require the wave-function to satisfy

$$
\begin{gathered}
\phi_{m_{1}, \ldots, m_{l}, \ldots, m_{k}, \ldots, m_{N}}\left(\mathbf{q}_{1}, \ldots, \mathbf{q}_{l}, \ldots, \mathbf{q}_{k}, \ldots, \mathbf{q}_{N}\right) \\
= \pm \phi_{m_{1}, \ldots, m_{k}, \ldots, m_{l}, \ldots, m_{N}}\left(\mathbf{q}_{1}, \ldots, \mathbf{q}_{k}, \ldots, \mathbf{q}_{l}, \ldots, \mathbf{q}_{N}\right)
\end{gathered}
$$

for the boson/fermionic cases.

\section{A field theoretic description of the non-Abelian Chern-Simons particles}

Motivated by the fact that the Abelian Schrödinger field describes Abelian charged particles when second quantized, one may guess that a non-Abelian second quantized Schrödinger field describes particles carrying non-Abelian charges. Since we are considering such particles in interaction with a non-Abelian gauge field, whose dynamics is governed by the ChernSimons term, the Lagrangian for the system should contain the Schrödinger Lagrangian and the Chern-Simons term with a minimal coupling between them.

Thus, consider the Lagrange density,

$$
\mathcal{L}=i \psi^{\dagger} D_{t} \psi-\frac{1}{2}(\mathbf{D} \psi)^{\dagger} \cdot(\mathbf{D} \psi)-\kappa \epsilon^{\alpha \beta \gamma} \operatorname{tr}\left(A_{\alpha} \partial_{\beta} A_{\gamma}+\frac{2}{3} A_{\alpha} A_{\beta} A_{\gamma}\right)
$$

where the covariant derivative $D_{\mu}$ is defined by

$$
D_{\mu} \equiv \partial_{\mu}+A_{\mu}
$$

and the mass has been scaled to unity. Also we assume that the Schrödinger fields is in a certain representation of the group generator, $T^{a}$. Let us record here the Euler-Lagrange equations.

$$
\begin{gathered}
i D_{t} \psi=-\frac{1}{2} \mathbf{D} \cdot \mathbf{D} \psi \\
\frac{\kappa}{2} \epsilon^{\alpha \beta \gamma} F_{\beta \gamma}=J^{\alpha}
\end{gathered}
$$


The non-Abelian charge density and spatial current density read

$$
\begin{aligned}
& J^{0} \equiv \rho=T_{a} \rho^{a}=-i T_{a}\left(\psi^{\dagger} T^{a} \psi\right) \\
& \mathbf{J}=-\frac{1}{2} T_{a}\left[\psi^{\dagger} T^{a} \mathbf{D} \psi-(\mathbf{D} \psi)^{\dagger} T^{a} \psi\right]
\end{aligned}
$$

which satisfy a covariant continuity equation (as a consequence of the Euler-Lagrange equation),

$$
\partial_{\mu} J^{\mu}+\left[A_{\mu}, J^{\mu}\right]=0
$$

Since the physical system in (33) is certainly constrained, we follow the symplectic methods of Hamiltonian reduction ${ }^{16}$ to find the required symplectic structure. For this purpose, we rewrite the Lagrange density (33) in canonical Darboux form with constraint,

$$
\mathcal{L}=i \psi^{\dagger} \dot{\psi}-2 \kappa \operatorname{tr} \dot{A}_{1} A_{2}-\frac{1}{2}(\mathbf{D} \psi)^{\dagger} \cdot(\mathbf{D} \psi)+2 \operatorname{tr} A_{0}(\kappa B+\rho)
$$

where irrelevant total derivative terms are dropped. It is clear from (39) that the Lagrange multiplier $A_{0}$, enforce the constraint

$$
B+\frac{\rho}{\kappa}=0
$$

It should be noted that Eq. (40) is of the same form as Eq. (14). Using definitions in (16), the constraint may again be solved by the relation (15). Inserting this solution into (39), we are left with

$$
\begin{aligned}
& \mathcal{L}=i \psi^{\dagger} \dot{\psi}+\kappa \dot{A}_{1 a} \int d^{2} r^{\prime} \mathcal{M}^{a}{ }_{b}\left(\mathbf{r}, \mathbf{r}^{\prime}\right)\left[\partial_{2}^{\prime} A_{1}^{b}\left(\mathbf{r}^{\prime}\right)+\frac{1}{\kappa} \rho^{b}\left(\mathbf{r}^{\prime}\right)\right] \\
& -\frac{1}{2}\left\{\left|\left(\partial_{1}+A_{1}\right) \psi\right|^{2}+\left|\left(\partial_{2}+\int d^{2} r^{\prime} T_{a} \mathcal{M}^{a}{ }_{b}\left(\mathbf{r}, \mathbf{r}^{\prime}\right)\left[\partial_{2}^{\prime} A_{1}^{b}\left(\mathbf{r}^{\prime}\right)+\frac{1}{\kappa} \rho^{b}\left(\mathbf{r}^{\prime}\right)\right]\right) \psi\right|^{2}\right\}
\end{aligned}
$$

While the constraint has been eliminated, the 1 -form in $\mathcal{L} d t$ is not canonical. To effect the Darboux transformation to canonical variables, let us rewrite (41) in terms of the transformed, primed, variables.

$$
\begin{aligned}
& \psi^{\prime}=U^{-1} \psi, \quad A_{1}^{\prime}=U^{-1} A_{1} U+U^{-1} \partial_{1} U \\
& A_{2}^{\prime}=U^{-1} \int d^{2} r^{\prime} T_{a} \mathcal{M}^{a}{ }_{b}\left(\mathbf{r}, \mathbf{r}^{\prime}\right)\left[\partial_{2}^{\prime} A_{1}^{b}\left(\mathbf{r}^{\prime}\right)+\frac{1}{\kappa} \rho^{b}\left(\mathbf{r}^{\prime}\right)\right] U+U^{-1} \partial_{2} U
\end{aligned}
$$

Then, the resulting Lagrangian is

$$
\begin{gathered}
\mathcal{L}=i \psi^{\prime \dagger} \dot{\psi}^{\prime}+\kappa\left(\dot{A}_{1}^{\prime a}\right) A^{\prime}{ }_{2 a}-\frac{1}{2}\left|\left(\nabla-\mathbf{A}^{\prime}\right) \psi^{\prime}\right|^{2} \\
-\frac{\kappa}{3} \int d^{2} r \epsilon^{\alpha \beta \gamma} \operatorname{tr} U^{-1} \partial_{\alpha} U U^{-1} \partial_{\beta} U U^{-1} \partial_{\gamma} U
\end{gathered}
$$


Here, we have used (40) and dropped unnecessary boundary terms for simplicity. Since (40) is transformed covariantly by (42), $A_{1}^{\prime}$ and $A_{2}^{\prime}$ satisfy the transform of relation (40),

$$
\partial_{1} A_{2}^{\prime}-\partial_{2} A_{1}^{\prime}+\left[A_{1}^{\prime}, A_{2}^{\prime}\right]-\frac{\rho^{\prime}}{\kappa}=0
$$

where $\rho^{\prime}$ denotes $-i T_{a} \psi^{\prime \dagger} T^{a} \psi^{\prime}$. Now, as in Section II, take $U$ to satisfy

$$
A_{1}^{\prime}=U^{-1} A_{1} U+U^{-1} \partial_{1} U=0
$$

Although Eq. (42) with $U$ solving (45), gives an explicit expression for $A_{2}^{\prime}$, it is more convenient to obtain the expression for $A_{2}^{\prime}$ from (44) (the $U$ should be consistently chosen): the solution of (44) with $A_{1}^{\prime}=0$ is simply

$$
A_{2}^{\prime}=\frac{1}{\kappa \partial_{1}} \rho^{\prime}
$$

Inserting (45) and (46) into (43), the desired Lagrange density is finally given by

$$
\mathcal{L}=i \psi^{\dagger} \dot{\psi}-\frac{1}{2}\left\{\left|\partial_{1} \psi\right|^{2}+\left|\left(\partial_{2}+\frac{1}{\kappa \partial_{1}} \rho\right) \psi\right|^{2}\right\}
$$

where the prime is dropped and the decoupled topological degree of freedom $\left[\operatorname{tr}\left(U^{-1} d U\right)^{3}\right]$ is omitted.

Upon quantization, the Lagrange density (47) provides a (bosonic) equal-time commutation relation.

$$
\left[\psi_{n}(\mathbf{r}), \psi_{m}^{\dagger}\left(\mathbf{r}^{\prime}\right)\right]=\delta_{n m} \delta\left(\mathbf{r}-\mathbf{r}^{\prime}\right)
$$

(For definiteness and simplicity, we take a bosonic algebra; a similar analysis can be easily given with a fermionic degree of freedom.) Then the Hamiltonian density for the system is given by

$$
\mathcal{H}=\frac{1}{2}\left\{\left|\partial_{1} \psi\right|^{2}+\left|\left[\partial_{2}+\frac{1}{\kappa} \int d^{2} r^{\prime} G\left(\mathbf{r}-\mathbf{r}^{\prime}\right) \rho\left(\mathbf{r}^{\prime}\right)\right] \psi\right|^{2}\right\}
$$

where $\frac{1}{\partial_{1}}$ is represented by $\int d^{2} r^{\prime} G\left(\mathbf{r}-\mathbf{r}^{\prime}\right)$ as in Section II.

To describe the Hamiltonian accurately, we begin by defining operator covariant derivative

$$
\mathbf{D} \psi=(\nabla-\mathbf{A}) \psi
$$

where A denotes

$$
\mathbf{A}=\frac{1}{\kappa} \int d^{2} r^{\prime} \mathbf{G}\left(\mathbf{r}-\mathbf{r}^{\prime}\right) \rho\left(\mathbf{r}^{\prime}\right)
$$

From the commutation relation, it follows that

$$
\left[A_{i a}(\mathbf{r})\left(T^{a}\right)_{m n}, \psi_{n}\left(\mathbf{r}^{\prime}\right)\right]=\frac{i}{\kappa} G_{i}\left(\mathbf{r}-\mathbf{r}^{\prime}\right)\left(T^{a} T_{a} \psi\left(\mathbf{r}^{\prime}\right)\right)_{m}
$$


Note that $\mathbf{G}(\mathbf{r})$ is ill defined at the origin. If we prescribe that $\mathbf{G}$ vanishes at the origin, $\psi(\mathbf{r})$ and $\psi^{\dagger}(\mathbf{r})$ commute with $\mathbf{A}(\mathbf{r})$ and so no ordering problem arises. It can be easily checked that also no ordering problem arises in relation with $\mathbf{A} \cdot \mathbf{A}$. With this prescription, the Hamiltonian for the system is

$$
H=\frac{1}{2} \int d^{2} r(\mathbf{D} \psi)^{\dagger} \cdot(\mathbf{D} \psi)
$$

The operator field equation of motion follows by commutation with $H$.

$$
\begin{aligned}
i \dot{\psi}(\mathbf{r}) & =[\psi(\mathbf{r}), H] \\
& =-\frac{1}{2} \mathbf{D} \cdot \mathbf{D} \psi(\mathbf{r})-i A_{0} \psi(\mathbf{r}) \\
& +\frac{1}{2 \kappa^{2}} \int d^{2} r^{\prime} \mathbf{G}\left(\mathbf{r}^{\prime}-\mathbf{r}\right) \cdot \mathbf{G}\left(\mathbf{r}^{\prime}-\mathbf{r}\right) \psi^{\dagger}\left(\mathbf{r}^{\prime}\right) T_{a} T_{b} \psi\left(\mathbf{r}^{\prime}\right) T^{a} T^{b} \psi(\mathbf{r}) \\
& \equiv \hat{O} \psi
\end{aligned}
$$

$A_{0}$ in (54) is given by

$$
A_{0}(\mathbf{r})=-\frac{1}{\kappa} \int d^{2} r^{\prime} \mathbf{G}\left(\mathbf{r}^{\prime}-\mathbf{r}\right) \cdot \mathbf{J}(\mathbf{r})
$$

where $\mathbf{J}$ is the non-Abelian current-density operator, which takes the same form as (37). [Eqs. (51)and (55) solve (36) when the covariant continuity equation (38) is used and ordering issues are ignored.] The last term in the second equality of (54) is a quantum correction arising from reordering.

Note that the number density operator $\rho_{N} \equiv \psi^{\dagger} \psi$ satisfies the usual continuity equation:

$$
\dot{\rho}_{N}=i\left[H, \rho_{N}\right]=-\nabla \cdot \mathbf{j}_{N}
$$

where $\mathbf{j}_{N}$ is the $\mathrm{U}(1)$ spatial current density:

$$
\mathbf{j}_{N}=\frac{1}{2 i}\left[\psi^{\dagger} \mathbf{D} \psi-(\mathbf{D} \psi)^{\dagger} \psi\right]
$$

Thus, the number operator $N=\int d^{2} r \rho_{N}(\mathbf{r})$ commutes with the Hamiltonian and also satisfies the algebra

$$
\left[N, \psi_{m}(\mathbf{r})\right]=-\psi_{m}(\mathbf{r}), \quad\left[N, \psi_{m}^{\dagger}(\mathbf{r})\right]=\psi_{m}^{\dagger}(\mathbf{r})
$$

Now it is a simple matter to construct the $\mathrm{N}$-particle state. The vacuum state $\mid 0>$ is annihilated by $\psi_{m}(\mathbf{r})$ :

$$
\psi(\mathbf{r})|0>=<0| \psi^{\dagger}(\mathbf{r})=0
$$

and, therefore, it is a zero-eigenvalue eigenstate for the both $\mathrm{N}$ and $\mathrm{H}$. 
We define the $N$-particle state by $N$ successive operations of $\psi_{m}(\mathbf{r})$ on the vacuum bra $<0 \mid$. In this way, we are led to the $N$-particle wave function,

$$
\phi_{m_{1}, \ldots, m_{N}}\left(\mathbf{r}_{1}, \ldots, \mathbf{r}_{N}\right)=<0\left|\psi_{m_{1}}\left(\mathbf{r}_{1}\right), \ldots, \psi_{m_{N}}\left(\mathbf{r}_{N}\right)\right| \Phi>
$$

where $\mid \Phi>$ is a general state and the $N$-particle amplitude is selected by projecting $|\Phi\rangle$ onto the $N$-particle state.

When one computes the time evolution of the Schrödinger wave function in (60) for $N=1$, one simply gets

$$
i \partial_{t} \phi_{m}(\mathbf{r})=<0\left|(\hat{O} \psi)_{m}\right| \Phi>=-\frac{1}{2} \nabla^{2} \phi_{m}(\mathbf{r})
$$

where (54) and (59) are used to get the second equality: specifically, one needs

$$
<0\left|(\hat{O} \psi)_{m}=-\frac{1}{2} \nabla^{2}<0\right| \psi_{m}
$$

Hence the one-particle problem is free-there are no self interactions.

For the two-particle Schrödinger equation, we begin with

$$
\begin{aligned}
& i \partial_{t} \phi_{m_{1} m_{2}}\left(\mathbf{r}_{1}, \mathbf{r}_{2}\right)=<0\left|(\hat{O} \psi)_{m_{1}}\left(\mathbf{r}_{1}\right) \psi_{m_{2}}\left(\mathbf{r}_{2}\right)+\psi_{m_{1}}\left(\mathbf{r}_{1}\right)(\hat{O} \psi)_{m_{2}}\left(\mathbf{r}_{2}\right)\right| \Phi> \\
& \quad=<0\left|(\hat{O} \psi)_{m_{1}}\left(\mathbf{r}_{1}\right) \psi_{m_{2}}\left(\mathbf{r}_{2}\right)+(\hat{O} \psi)_{m_{2}}\left(\mathbf{r}_{2}\right) \psi_{m_{1}}\left(\mathbf{r}_{1}\right)+\left[\psi_{m_{1}}\left(\mathbf{r}_{1}\right),(\hat{O} \psi)_{m_{2}}\left(\mathbf{r}_{2}\right)\right]\right| \Phi>
\end{aligned}
$$

Upon using (62) and computing of the commutator $\left[\psi_{m_{1}}\left(\mathbf{r}_{1}\right),\left(\hat{O} \psi\left(\mathbf{r}_{2}\right)\right)_{m_{2}}\right]$, one finds the two particle Schrödinger equation to be

$$
i \partial_{t} \phi\left(\mathbf{r}_{1}, \mathbf{r}_{2}\right)=-\frac{1}{2}\left[\left(\nabla_{1}+\frac{i}{\kappa} \mathbf{G}\left(\mathbf{r}_{1}-\mathbf{r}_{2}\right) T_{1}^{a} T_{2 a}\right)^{2}+\left(\nabla_{2}+\frac{i}{\kappa} \mathbf{G}\left(\mathbf{r}_{2}-\mathbf{r}_{1}\right) T_{2}^{a} T_{1 a}\right)^{2}\right] \phi\left(\mathbf{r}_{1}, \mathbf{r}_{2}\right)
$$

where the operator $T_{k}^{a} \phi$ is defined by

$$
\left(T_{k}^{a} \phi\right)_{m_{1}, \ldots, m_{k}, \ldots, m_{N}}=\left(T_{k}^{a}\right)_{m_{k} m_{k}^{\prime}} \phi_{m_{1}, \ldots, m_{k}^{\prime}, \ldots, m_{N}}
$$

By a similiar straightforward computation, the $N$-body Schrödinger equation is

$$
\begin{aligned}
i \partial_{t} \phi\left(\mathbf{r}_{1}, \ldots, \mathbf{r}_{N}\right) & =H_{N} \phi\left(\mathbf{r}_{1}, \ldots, \mathbf{r}_{N}\right) \\
& =-\frac{1}{2} \sum_{k}\left[\nabla_{k}+\frac{i}{\kappa} \sum_{l \neq k} \mathbf{G}\left(\mathbf{r}_{k}-\mathbf{r}_{l}\right) T_{k}^{a} T_{l a}\right]^{2} \phi\left(\mathbf{r}_{1}, \ldots, \mathbf{r}_{N}\right)
\end{aligned}
$$

which coincides with (31), for identical bosons with their common mass scaled to unity $\left(T_{k}^{a}\right.$ corresponds to $\left.i I_{k}^{a}\right)$. 


\section{Interpretation of the connection $\mathcal{G}_{k}$}

The connection $\mathcal{G}_{k}(27)$ involves the delta function (26); in the Schrödinger equation (31) or (66) it occurs squared, which requires well-definition. It is known how to deal with this problem. ${ }^{18}$ First, regularize the delta function in terms of a peaked function of width $l$ and height $o\left(\frac{1}{l}\right)$. Then, equipped with this regulated delta function, solve the Schrödinger equation within a small region where the connection does not vanish. Finally by a limiting procedure, which takes $l$ to zero, one arrives at a boundary condition for the wave function (replacing the set of points on which the connection is singular). Once the above procedure is implemented, one finds that the 2-body Schrödinger equation is equivalent to

$$
i \partial_{t} \phi\left(\mathbf{r}_{1}, \mathbf{r}_{2}\right)=-\frac{1}{2}\left(\nabla_{1}^{2}+\nabla_{2}^{2}\right) \phi\left(\mathbf{r}_{1}, \mathbf{r}_{2}\right)
$$

for $y_{1} \neq y_{2}$ with a cut at $y_{1}=y_{2} \equiv y$. On the cut the boundary condition is

$$
\begin{aligned}
& \phi\left(\mathbf{r}_{1}=\left(x_{1}, y+0^{+}\right), \mathbf{r}_{2}=\left(x_{2}, y\right)\right) \\
& =e^{\frac{i}{2 \kappa} \epsilon\left(x_{1}-x_{2}\right) T_{1}^{a} T_{2 a}} \phi\left(\mathbf{r}_{1}=\left(x_{1}, y+0^{-}\right), \mathbf{r}_{2}=\left(x_{2}, y\right)\right)
\end{aligned}
$$

The $N$-body equation becomes

$$
i \partial_{t} \phi\left(\mathbf{r}_{1}, \ldots, \mathbf{r}_{N}\right)=-\frac{1}{2} \sum \nabla_{k}^{2} \phi\left(\mathbf{r}_{1}, \ldots, \mathbf{r}_{N}\right) \text { for } y_{k} \neq y_{l}(k \neq l)
$$

with boundary conditions on the cut $y_{k}=y_{l} \equiv y_{k l}(k \neq l)$

$$
\begin{aligned}
& \phi\left(\mathbf{r}_{1}, \ldots, \mathbf{r}_{k}=\left(x_{k}, y_{k l}+0^{+}\right), \ldots, \mathbf{r}_{l}=\left(x_{l}, y_{k l}\right), \ldots, \mathbf{r}_{N}\right) \\
& =e^{\frac{i}{2 \kappa} \epsilon\left(x_{k}-x_{l}\right) T_{k}^{a} T_{l a} \phi}\left(\mathbf{r}_{1}, \ldots, \mathbf{r}_{k}=\left(x_{k}, y_{k l}+0^{-}\right), \ldots, \mathbf{r}_{l}=\left(x_{l}, y_{k l}\right), \ldots, \mathbf{r}_{N}\right)
\end{aligned}
$$

As claimed in Section II, the Green's function $G$ in (23) is restricted by the consistency of the Schrödinger equation. If we choose, for example, $G$ as

$$
G(\mathbf{r})=\frac{1}{2} \delta(y)(\epsilon(x)+C) \quad(C \neq 0)
$$

the resulting 2-body Schrödinger equation reads

$$
i \partial_{t} \phi\left(\mathbf{r}_{1}, \mathbf{r}_{2}\right)=-\frac{1}{2}\left[\begin{array}{c}
\left(\partial_{1}^{x}\right)^{2}+\left(\partial_{2}^{x}\right)^{2} \\
+\left(\partial_{1}^{y}-\frac{i}{2 \kappa} \delta\left(y_{1}-y_{2}\right)\left(\epsilon\left(x_{1}-x_{2}\right)+C\right) T_{1}^{a} T_{2 a}\right)^{2} \\
+\left(\partial_{2}^{y}-\frac{i}{2 \kappa} \delta\left(y_{2}-y_{1}\right)\left(\epsilon\left(x_{2}-x_{1}\right)+C\right) T_{2}^{a} T_{1 a}\right)^{2}
\end{array}\right] \phi\left(\mathbf{r}_{1}, \mathbf{r}_{2}\right)
$$


Since $\epsilon\left(x_{1}-x_{2}\right)+C \neq-\left(\epsilon\left(x_{2}-x_{1}\right)+C\right)$, inconsistent boundary conditions are obtained on the cut. Moreover, for $C \neq 0 \mathrm{Eq}$. (72) is not Galileo-invariant. [It should be noted that the boundary condition in (70) is consistent with the exchange-symmertry of the wave function. An equivalent boundary condition is postulated in Ref. [19].]

In Refs. [13] and [14], there appears a Schrödinger equation where the potential is the complex Knizhnik-Zamolodchikov connection.

$$
\begin{gathered}
i \partial_{t} \phi^{\prime}=H_{N}^{\prime} \phi^{\prime} \\
H_{N}^{\prime}=-\frac{1}{2} \sum_{k}\left[\nabla_{k}+\frac{i}{\kappa} \sum_{l \neq k} \mathbf{G}_{K Z}\left(\mathbf{r}_{k}-\mathbf{r}_{l}\right) T_{k}^{a} T_{k a}\right]^{2} \\
\mathbf{G}_{K Z}(\mathbf{r})=\frac{1}{2 \pi(x+i y)}\left(\begin{array}{c}
i \\
-1
\end{array}\right)
\end{gathered}
$$

[Notation here is changed from complex coordinates to real $(x, y)$ coordinates.] To show that the equation in (73) is equivalent to $(31)$ or (66), we must find a time-independent $V$ that connects these equations.

$$
\phi^{\prime}=V \phi, \quad H_{N}^{\prime}=V H_{N} V^{-1}
$$

Eq. (75) holds when $V$ satisfies

$$
V^{-1}\left[\nabla_{k}+\frac{i}{\kappa} \sum_{l \neq k} \mathbf{G}_{K Z}\left(\mathbf{r}_{k}-\mathbf{r}_{l}\right) T_{k}^{a} T_{l a}\right] V=\frac{i}{\kappa} \sum_{l \neq k} \mathbf{G}\left(\mathbf{r}_{k}-\mathbf{r}_{l}\right) T_{k}^{a} T_{l a}
$$

For the two particle case, this is solved by

$$
\begin{aligned}
& V=e^{\frac{1}{2 \pi \kappa} a\left(\mathbf{r}_{1}-\mathbf{r}_{2}\right) T_{1}^{a} T_{2 a}} \\
& a(\mathbf{r})=\ln r-i \tan ^{-1}\left|\frac{x}{y}\right| \epsilon(x) \epsilon(y)+i \frac{\pi}{2}
\end{aligned}
$$

where $\tan ^{-1} x$ lies in $\left[0, \frac{\pi}{2}\right]$ for $x \geq 0$. It should be noted that $V$ is a single-valued function of $\mathbf{r}_{1}$ and $\mathbf{r}_{2}$ and multi-valuedness does not arise. In addition, the transformation respects the exchange symmetry of the wave function since $V\left(\mathbf{r}_{1}, \mathbf{r}_{2}\right)=V\left(\mathbf{r}_{2}, \mathbf{r}_{1}\right)$. Solving (76) for general $N$, remains an open problem.

We have not here included a non-Abelian point interaction, which presumably would protect the conformal invariance of the model in field theoretical perturbation theory, just as it does in the Abelian model. ${ }^{11}$ This topic is under further investigation. ${ }^{20}$ 


\section{APPENDIX}

The purpose of this Appendix is to provide a symplectic structure for the non-Abelian charge $I$. While this problem is analyzed in many places, ${ }^{21}$ the group is restricted to $S O(n), S U(n)$ or especially $S U(2)$, and the discussion is quite involved since explicit Darboux variables are constructed before canonical commutators are found. Here we give a simple derivation for a general group without passing to canonical Darboux variables. (The only required condition is that the Lie algebra possesses a nonsingular metric.) As stated in Section II, the symplectic structure for the non-Abelian charge $I$ is given by $2 \operatorname{tr} K g^{-1} \dot{g}$, where $I=g K g^{-1}$ and $K$ is time independent.

Let us take part of (7) to describe the dynamics of $I$. Namely, consider the Lagrangian

$$
L_{I}=2 \operatorname{tr} K g^{-1} \dot{g}+2 \operatorname{tr} I A
$$

For this portion of the complete problem, $A$ is viewed as "external". When the Lagrangian is varied with respect to $g$, the Euler-Lagrange equation is

$$
\dot{I}+[A, I]=0
$$

To find the classical phase space, parametrize the group element $g$ with coordinates $\theta^{a}$ where index $a$ ranges over the dimension of the group, which coincides with the number of generators. The Lagrangian $L_{I}$ may be rewritten in terms of $\theta^{a}$.

$$
L_{I}=a_{a}(\theta) \dot{\theta}^{a}-I^{a} A_{a}
$$

It is convenient to express $a_{a}(\theta)$ as

$$
a_{a}=-C_{a}{ }^{b} I_{b}
$$

where the invertible matrix $C_{a}{ }^{b}$ is defined by the relation; $C_{a}{ }^{b} T_{b}=\partial_{a} g g^{-1}$. The symplectic two form $\omega_{a b} \equiv \partial_{a} a_{b}-\partial_{b} a_{a}$ may be computed using (A.4) and the definition $C_{a}{ }^{b}$ to give

$$
\omega_{a b}=C_{a}{ }^{c} C_{b}{ }^{d} I^{e} f_{c d e}
$$

which is not invertible since there are zero modes $p_{i}^{a}$.

$$
p_{i}^{a} \omega_{a b}=0
$$

[In fact we do not meet the inverse for $\omega_{a b}$ in out final formula (A.13), but it arises at intermediate steps in our derivation.]

If we use a projection operator $P_{a}{ }^{b}$ that satisfies

$$
P_{a}{ }^{b} P_{b}{ }^{c}=P_{a}{ }^{c}, \quad P_{a}{ }^{b} \omega_{b c}=0
$$


whose rank coincides with the number of zero modes, it is possible to find an inverse of $\omega_{a b}$ in the projected subspace. Namely, the inverse $\omega^{a b}$ is uniquely defined by the relations

$$
\begin{aligned}
& \omega_{a b} \omega^{b c}=\delta_{a}{ }^{c}-P_{a}{ }^{c} \\
& \omega^{a b}=-\omega^{b a}, \quad \omega^{a b} P_{b}{ }^{c}=0
\end{aligned}
$$

Once $\omega^{a b}$ is constructed, we give the fundamental Poisson bracket between functions $W_{l}$ of the $\theta$ as

$$
\left\{W_{1}, W_{2}\right\}=\partial_{a} W_{1} \omega^{a b} \partial_{b} W_{2}
$$

It can be easily checked that the Jacobi identity

$$
\left\{W_{1},\left\{W_{2}, W_{3}\right\}\right\}+\left\{W_{2},\left\{W_{3}, W_{1}\right\}\right\}+\left\{W_{3},\left\{W_{1}, W_{2}\right\}\right\}=0
$$

is insured when

$$
P_{a}^{b} \partial_{b} W_{l}=0
$$

Hence we use the bracket (A.9) only between function $W_{l}$ of $\theta^{a}$ that satisfy (A.11).

We now turn to the problem of calculating the bracket between generators $I$. First noting that

$$
\partial_{m} I_{a}=\left(C^{-1}\right)_{a}{ }^{b} \omega_{b m}
$$

it follows from (A.7) that (A.11) is satisfied by I. Moreover, using (A.5,7,8,12), we immediately conclude that $I$ satisfies the expected bracket algebra.

$$
\left\{I_{a}, I_{b}\right\}=\partial_{m} I_{a} \omega^{m n} \partial_{n} I_{b}=-f_{a b}{ }^{c} I_{c}
$$

In this derivation, explicit expressions of the zero modes and the projection operator are not used. It may be interesting to obtain them. To find the zero modes explicitly, first construct all Lie algebra elements $K_{i}^{a} T_{a}$ that solve

$$
f_{a b c} K_{i}^{b} K^{c}=0
$$

[The number of such Lie algebra elements is greater than or equal to the rank of the Lie algebra.] Then, define $p_{i}^{a}$ through relations,

$$
I_{i}^{a} T_{a}=g K_{i} g^{-1}, \quad p_{i}^{a}=I_{i}^{b}\left(C^{-1}\right)_{b}^{a}
$$

and it is simple matter to show that the $p_{i}$ satisfy (A.6). Now let us construct dual vector $\bar{p}_{i a}$ in the following way. First define a coordinate transformation from the $\theta^{a}$ coordinates to new coordinates $\left(\xi_{1}, \ldots, \xi_{k}, \eta_{1}, \ldots, \eta_{d-k}\right)$ (where $k$ and $d$ are respectively the number of zero modes and the dimension of the group manifold), such that

$$
\frac{\partial \theta^{a}}{\partial \xi_{i}}=p_{i}^{a}
$$


Then, $\bar{p}_{i}$ is defined by

$$
\bar{p}_{i a} \equiv \frac{\partial \xi_{i}}{\partial \theta^{a}}
$$

and by construction, they satisfy

$$
\bar{p}_{i a} p_{j}^{a}=\delta_{i j}
$$

Notice $\bar{p}_{i a} p_{i}^{b}$ satisfies (A.7) and its rank is $k$. Hence it may serve as projection operator in (A.8).

We prove that the Lagrangion (A.1) does not depend on the $\xi_{i}$ variables except for total derivatives. To show this, consider an infinitesimal transformation

$$
\delta_{\epsilon} \theta^{a}=\epsilon(t) p^{a}
$$

Then the variation of the Lagrangian is

$$
\delta_{\epsilon} L_{I}=\frac{d}{d t}\left(\epsilon p_{i}^{a} a_{a}\right)=\frac{d}{d t}\left(-\epsilon K_{i}^{a} K_{a}\right)=\delta_{\epsilon} \frac{d}{d t}\left(-\xi_{i} K_{i}^{a} K_{a}\right)
$$

To get the second equality in (A.20), we use (A.4) and (A.15). Noting

$$
\delta_{\epsilon}\left(L_{I}+\dot{\xi}_{i} K_{i}^{a} K_{a}\right)=0
$$

we conclude that $L_{I}+\dot{\xi}_{i} K_{i}^{a} K_{a}$ does not depend on $\xi_{i}$. Hence, when we transform to the new coordinates $(\xi, \eta)$, only the $\eta$ variables are relevant for the classical phase space and the $\xi$ variables may be dropped from the Lagrangian.

Finally we remark that explicit Darboux variables for the $S U(n)$ and $S O(n)$ groups have been found, and especially the case of $S U(2) \sim S O(3)$ is well known. ${ }^{21}$ Let us record an alternate expression for the $S O(3)$ canonical 1-form, which also serves to quantize the isospin algebra.

$$
L_{I}^{\prime}=\mathbf{A}(\mathbf{I}) \cdot \dot{\mathbf{I}}
$$

Here $\mathbf{A}(\mathbf{r})$ is the potential for the Dirac point monopole of strength $-|g|$ and $\mathbf{I}$ is restricted to the surface of a sphere of radius $|g|$. Even though the Dirac monopole defines the singular 2-form, $F_{i j}(\mathbf{r})=-\epsilon^{i j k} \frac{r^{k}}{g^{2}}$, this possess an inverse on the tangent space of the sphere, and in this way we obtain $\left\{I^{i}, I^{j}\right\}=-\epsilon^{i j k} I^{k}$. 


\section{REFERENCES}

1. S. K. Wong, Nuovo Cimento 65 A (1970) 689.

2. C. Hagen, Ann. Phys. (NY) 157 (1984) 342, Phys. Rev. D 31 (1985) 2135.

3. J. Leinaas and J. Myrheim, Nuovo Cimento 37 B (1977) 1.

4. F. Wilczek, Phys. Rev. Lett. 49 (1982) 957.

5. The Quantum Hall Effect, R. Prange and S. Girvin, eds., (Springer, Berlin, 1990).

6. R. B. Laughlin, Phys. Rev. Lett. 60 (1988) 2677.

7. R. Jackiw, Ann. Phys. (NY) 201 (1990) 83.

8. R. Jackiw and S.-Y. Pi, Phys. Rev. D 42 (1990) 3500, (E) 48 (1993) 3929.

9. R. Jackiw and S.-Y. Pi, Phys. Rev. Lett. 64 (1990) 2669, (C) 66 (1991) 2682.

10. R. Jackiw and S.-Y. Pi, Nucl. Phys. B (Proc. Suppl.) 33C (1993) 104.

11. G. Lozano, Phys. Lett. B 283 (1992) 70; O. Bergman and G. Lozano, Ann. Phys. (NY) 229 (1994) 416; D. Freedman, G. Lozano and N. Rius, Phys. Rev. D 49 (1994) 1054; G. Amelino-Camelina, Phys. Lett. B (in press).

12. G. Dunne, R. Jackiw, S.-Y. Pi and C. A. Trugenberger, Phys. Rev. D 43 (1991) 1332, (E) 45 (1992) 3012; G. Dunne, Comm. Math. Phys. 150 (1993) 519.

13. E. Verlinde in Modern Quantum Field Theory, edited by S. Das et al. (World Scientific, Singapore, 1991).

14. T. Lee and P. Oh, Ann. Phys. (NY) (in press), Phys. Lett. B 319 (1993) 497.

15. V. Knizhnik and A. Zamolodchikov, Nucl. Phys. B 247 (1984) 83.

16. L. Faddeev and R. Jackiw, Phys. Rev. Lett. 60 (1988) 1692.

17. S. Deser, R. Jackiw and S. Templeton, Phys. Rev. Lett. 48 (1982) 975, Ann. Phys. (NY) 140 (1982) 372, (E) 185 (1988) 406.

18. A. Kapustin and P. Pronin, Phys. Lett. B 303 (1993) 45.

19. H. Lo and J. Preskill, Califonia Institute of Technology preprint (1993) CALT-68-1867.

20. D. Bak and O. Bergman, in preparation.

21. H. B. Nielsen, D. Rohrlich, Nucl. Phys. B 299 (1988) 471; A. Alekseev, L. Faddeev and S. Shatashvili, J. Geom. Phys. 3 (1989) 1; K. Johnson, Ann. Phys. (NY) 192 (1989) 104; A. Balachandran, G. Marmo, B. Skagerstam and A. Stern, Classical Topology and Quantum States (World Scientific, Singapore, 1991). 